\title{
Laparoscopic biliary surgery: will we ever learn?
}

\author{
Jeffrey L. Ponsky
}

Published online: 24 August 2010

(C) Springer Science+Business Media, LLC 2010

The Guideline for laparoscopic biliary surgery appearing in this issue of Surgical Endoscopy is comprehensive and accurate. It reflects the extensive experience of the authors and a realistic view of practice patterns in the USA. There can be no doubt that laparoscopic cholecystectomy, in all but the most difficult cases or where neoplasia is suspected or exists, has become the standard of care for disease of the gallbladder. Carefully practiced, it provides excellent relief of symptoms with minimal morbidity and is a great advance over the former open approach. The impact of laparoscopic surgery upon the management of common bile duct stones is not at all so clear. Indeed, it may be argued that the issue has become confused.

Forty years ago, bile duct stones, regardless of their presentation, were managed only with open choledocholithotomy. When the gallbladder was present, it was removed. The ensuing decades and the development of endoscopic cholangiography with sphincterotomy and laparoscopic cholecystectomy have provided physicians and surgeons with multiple options and alternatives in their care of patients. It must be remembered, however, that open choledocholithotomy is the standard against which newer approaches must be measured.

We have learned that routine preoperative endoscopic retrograde cholangiopancreatography (ERCP) is costly and unnecessary. It also adds significant potential for morbidity. Even when stones are more likely to exist, as in patients with recent pancreatitis or a dilated common bile duct noted on ultrasound, preoperative ERCP is often negative and unnecessary. In these patients, laparoscopic cholecystectomy with

\section{J. L. Ponsky $(\bowtie)$}

Department of Surgery, University Hospitals of Cleveland,

Case Western Reserve University and University Hospitals, Cleveland, OH, USA

e-mail: jponsky@yahoo.com intraoperative cholangiogram should be performed. If stones are found, they may be managed by laparoscopic extraction techniques, by conversion to open common bile duct exploration, or by postoperative ERCP. Only when there is significant jaundice is preoperative ERCP imperative to rule out an impacted stone or tumor.

Laparoscopic clearance of the common duct, while well described, is infrequently practiced. Appreciation of common duct stones and their clearance depend upon use of operative cholangiography, which is itself infrequently practiced. Techniques of biliary exploration such as transcystic bile duct exploration, choledochoscopy, and laparoscopic choledochotomy are rarely performed. Most stones are relegated to postoperative ERCP if they are recognized. That has become the most common clinical scenario and works well in most cases. However, a generation of surgeons has emerged from training with little experience in bile duct surgery. Common bile duct exploration, by any means, is rarely performed.

In the future, we must identify means to train our surgeons, residents, and those in practice in the techniques of bile duct exploration. This should include endoscopic, laparoscopic, and open methods. The result will be the most efficacious, cost-efficient, and safe therapy for our patients.

\section{Selected reading}

1. Rhodes M, Sussman L, Cohen L, Lewis M (1998) Randomised trial of laparoscopic exploration of common bile duct versus postoperative endoscopic retrograde cholangiography for common bile duct stones. Lancet 351(9097):159-161

2. Rogers SJ, Cello JP, Horn JK, Siperstein AE, Schecter WP, Campbell AR, Mackersie RC, Rodas A, Kreuwel HT, Harris HW (2010) Prospective randomized trial of LC + LCBDE vs ERCP/ $\mathrm{S}+\mathrm{LC}$ for common bile duct stone disease. Arch Surg 145(1): $28-33$ 